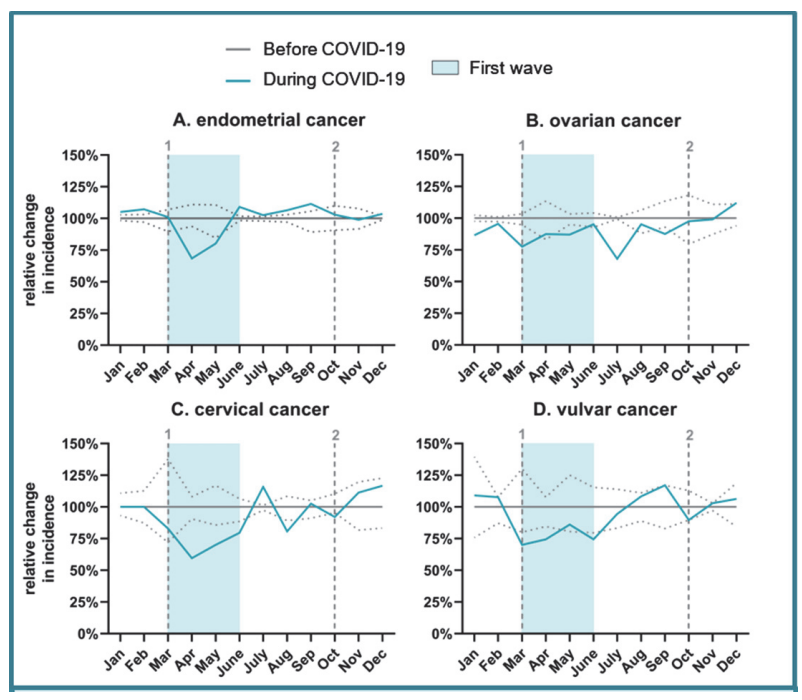

Abstract 243 Figure 1 Change in incidence over time

Introduction/Background* On the 11th of March 2020, the novel severe acute respiratory syndrome corona virus 2 (SARS-COV-2) was declared a pandemic. We studied the incidence and missed diagnoses in gynaecological oncology as a result of the impact of the COVID-19 pandemic and consequent lockdown and overcrowded hospitals in the Netherlands.

Methodology We performed a retrospective cohort study using data from the Netherlands Cancer Registry (NCR) on women of 18 years and older diagnosed with invasive endometrial, ovarian, cervical or vulvar cancer in the period 2017-2020. The incidence was calculated for the period before the COVID-19 pandemic (mean number of the period 2017-2019) and compared with the incidence during the COVID-19 pandemic (2020) for each type of gynaecological cancer. The number of missed diagnoses was calculated as the difference in incidence between the period before and during the pandemic. Analyses were stratified for age, socioeconomical status (SES) and region.

Result(s)* The incidence rate of gynaecological cancer was 57/100.000 ( $\mathrm{n}=4921)$ before and 53/100.000 ( $\mathrm{n}=4682)$ during the pandemic. Comparing the incidence of the two periods for the four types of cancer showed no significant difference $\left(c h i^{2} \mathrm{p}=0.23\right)$. During the first wave of COVID19 (March-June 2020), a clear decrease in incidence was visible for all four types of gynaecological cancer (figure 1). Subsequently, large increases in incidence were visible. A total of 299 diagnoses were missed during the pandemic (6.2\% of the incidence of the period before COVID-19). The largest number of missed diagnoses was observed in ovarian cancer ( $\mathrm{n}=157,10.9 \%)$, followed by cervical cancer $(8.3 \%, \mathrm{n}=76)$, vulvar cancer $(7.1 \%, \mathrm{n}=31)$ and endometrial cancer $(1.7 \%, \mathrm{n}=35)$. No significant differences in incidence were found for different age groups, SES and between regions.

Conclusion* In the Netherlands, a clear drop in incidence was visible for all four types of gynaecological cancers during the first wave, which was caught up in the second part of 2020 hence no significant difference with the period before COVID-19. The difference in incidence was not caught up completely, for 299 women walk around with missed diagnoses, which could result in higher stage disease or even worse.

\section{CONSTRUCTION OF PRE-OPERATIVE PREDICTION MODEL AND ITS USE IN GYNAECOLOGICAL ONCOLOGY USING CARDIOPULMONARY EXERCISE TESTING AND ROUTINE HEALTH DATA}

\author{
${ }^{1 ; 2} \mathrm{NL}$ Whitham*${ }^{*}{ }^{1 ; 2} \mathrm{~J}$ Knight, ${ }^{2} \mathrm{~N}$ Wood. 'Lancaster University, Bailrigg, UK; ${ }^{2}$ Royal Preston \\ Hospital, Fulwood, UK
}

10.1136/ijgc-2021-ESG0.257

Introduction/Background* Our ever-growing population is putting greater strain upon the NHS with more complex medical problems, and strained resources. Comorbidities predispose patients to postoperative complications, impacting recovery and survival, length of stay, and mortality rates. The one size fits all attitude to treatment is no longer the best approach to tackle illness, as technology develops patient care will transform.

CPET assesses the bodies neurohumoral stress response to surgery, however not all patients complete CPET, with mobility and contraindications an issue. The ability to quantify morbidity and mortality risks enables discussions regarding appropriateness of surgical interventions, discuss likely scenarios and quality of life (QOL).

Methodology The aim is to create a pre-operative prediction model using cardiopulmonary exercise testing (CPET) and routine health data (RHD). The model can be utilised in conjunction with CPET, identifying patients in greater need of high dependency care (HDU), and at greater risk of complications.

All gynaecological oncology patients undergoing CPET from 2011 onwards are included in the retrospective analysis in one centre, which includes those over 65 years and those with multiple comorbidities. RHD, and CPET data will be collated, assessing links between the data with known clinical outcomes, producing a risk prediction tool that will then be used on a prospective cohort of patients.

Result(s)* Risk stratification tools allow shared decision making with personalised perioperative risks giving better patient experience and post-operative QOL.

RHD and CPET is currently being collated and analysed using R Studio.

Conclusion* The hope is to create a prediction model to use in conjunction with CPET to better guide care and improve patient outcome. If shown to better predict high risk patients it may be possible to improve care by prediction model alone, meaning all patients can be assessed, be cost effective, and be a more personalised approach to patient care. 